\title{
أزمة الهوية
}

\section{بين السياسات القومية وتتول دور الدولة}

\section{رضوان جودت زيادة*}

\section{مقدمة}

إذا كان سؤال الهوية هو السؤال الأكثر حضوراً في عصر العولمة، كوها متهمة بالسعي إلى تمديد الهوية، والعمل على إزالتها، فإن مفهوم الهوية نفسه يظل ملتبساً وعصيّاً على التحديد، بَلْه المقاربة. هذا اللَبس الذي ارتبط بالمفهوم نابعٌ من كونه يتداخل مع حقولٍ معرفية مختلفة، بحيث يصعب تجريده وتويله إلى مفهوٍٍ مكوِّن. إن الهوية بتداخلاكَا الانتربولوجية والسوسيولوجية والسياسية والثقافية، تشكل مفهوماً مكوَّاً من التداخلات المعرفية للحقول السابقة جميعها، بحكم كوغا رهينة بالمتن الاجتماعي وبالصيرورة التاريخية، ومن الصعب عليها الانفكاك منهما.

لذلك سنسعى في هذا البحث إلى دراسة معنى الهوية بحد ذاته، وهل هناك وجودٌ بجردٌ للهوية أم حضورٌ لخطاب الهوية؟ كما سنبحث في علاقة الهوية بالعولمة والثقافة، وانعكاسات هذا المفهوم المستجد للهوية على سلى الحقل السياسي، سيما فيما يتعلق بمفهوم الدولة-الأمة على ضوء التطور التاريخي للمجتمعات الغربية، التي يمكن القول إفا مرت بفترات تحقيب تاريخية واضحة، كالانتقال من الدولة-الأمة إلى دولة الحق والقانون أو الدولة الديمقراطية، بينما بقي هذا التطور داخل المجتمعات العربية أو الإسلامية غائماً، بحكم أن نموذج الدولة-الأمة نفسه داخل هذه المجتمعات لم يكن متبلوراً أو واضحاً تماماً.

\section{أولاً: إشكالية الموية وخطاب الهوية:}

إذا كان خطاب الهوية قد ورث خطاب القومية، بمعنى أن خطاب الهوية الثقافية قد حلَّ تدريجياً محل خطاب التحرير القومي الذي شاع إبّان مرحلة التحرر من الاستعمار، فإنه لدى بعض الباحثين ليس إلا شكلاً جديداً للقومية يعبر عن نفسه بتعابير التناقض الأيديولوجي أكثر مما يعبر بتعابير التناقض السياسي، فالشعوب التي كانت مستعمرة تعاني في الواقع الحالي عواقب عصرنة تمدد القيم الخاصة بها التي بُنيَ عليها سابقاً الإجماع ضد المحتل، وعلى ذلك علينا أن نتساءل لماذا بدأ الكلام عن الهوية في لحظة معينة، وليس في 
لحظة أخرى؟ وفي أي سياق تاريخي واجتماعي ظهر هذا الأمر؟ فالثقافة تأخذ أسبقية على السياسة، إذلم يسبق لنا أن تحدثنا عن الهوية أبداً بقدر ما نتحدث عنها اليوم؛ وذلك لأن الهويات باتت مهددة أكثر فأكثر، إن لم تكن مدمرة، إذ لا يُطالب الشعب بهويته إلا عندما يبدأ بالشك في وجودها، وعلى حد تعبير (جان ماري بونوا) "هناك وسواس يسود عصرنا الذي أشبع تواصلاً، إنه وسواس انطواء كل منا وانعزاله في أرضه، أي فيما يمقق اختلافه وأعني: هويته الخاصة، إنه الحلم بالتجذر في فضاءٍ منعزل." لكن، إذا كان الكلام عن الهوية حديث العهد، فلماذا يتم دائماً استحضار الهوية كتشكلٍ في الماضي، على اختلاف مفاهيمها، سواءً أكانت هوية ثقافية أم قومية أم هوية اثنية؟ إن الهوية بحسب هذا الخطاب تصبح مفهوماً مكتملاً وناجزاً، وليس علينا سوى البحث عن صيغة التطابق مع المثل (الماضي) لتحقق الوجود (الحاضر). وهذا ما دعا بعض الباحثين إلى التمييز بين الموية وخطاب الهوية، فإذا كانت الأولى حقيقة رمزية -على الأقل- تعيشها المجتمعات والأفراد فإن الخطاب المنشَأ عن الموية هو خطاب أيديولوجي ينصب تحت استراتيجيات العمل السياسي وتحولاته. فالهوية بوصفها ثقافة تشكل لحمة العمل الاجتماعي الشعبي، وهي بهذا المعنى تختلف عن الخصوصية التي هي محض خطاب أيديولوجي يتجه نحو الآخر بغية تأكيد الذات ورفض تماهيها مع الآخر ومع ما يطرحه، إنما سياسة الممانعة التي تتخذ صبغة ثقافية. وإذا كانت مسألة الآخر مكوّنة للهوية؛ فذلك لأن كل معرفٍة للذات هي بالضرورة مشوبة ومختلطة بنفي الآخر بغية توكيد الذات، فكنه الذات لا يتم إلا عن طريق الآخر ومن خلاله. فخطاب الهوية يطرح نفسه بوصفه خصوصية، على المجتمع في صيرورته أن يحافظ عليها، وعلى الآخر ألا يهددها أو يعمل على اختراقها، فتصبح الهوية بذلك بنيةً مصمتة غير قادرة على التواصل والمثاقفة، على اعتبار أن الآخر دوماً هو راغبّ في محو ثقافتها، وجادُ في العمل على تغيير حضارةما ومعالم وجودها. يرى (جان فرانسوا بايار) أن الهوية لا تعدو سوى أن تكون وهماً، فليست هناك سوى استراتيجيات للهوية يتبعها محترفو السياسية. فالعالم يعيش الآن قلقاً عاماً متعلقاً بالهوية، فهو يخشى توحيد الأشكال، ويخاف من تلاشي التمايزات، إذ إن هناك حركة عامة رامية إلى إزالة الحواجز بين المجتمعات بالعولمة مصحوبة باحتدام وتأجج الهويات الخاصة، سواءً أكانت عقائدية، أو قومية، أو إثنية. ولذلك تغدو العلاقة الجدلية بين الاتحاه نغو الكونية وتأكيد الخصوصيات حاضرة وراء أغلب الظواهر، التي تحتل مركز الصدارة في مختلف وسائل 
الإعلام من التوسع في الاقتصاد إلى رواج الفكر الديمقراطي خارج العالم الغربي، وانتشار الإعلام بشكلٍ غير مسبوق، وجموح موجات الهجرة إلى غير ذلك، ولكن السؤال الذي يجمع كل هذه التساؤلات، كيف يمكن أن نتفهم العلاقات بين الثقافة والسياسة دون الوقوع في فخ الانغلاق الثقافي؟ إذا اعتمدنا على (ماكس فيبر) في تعريفه للهوية، ذلك أن (فيبر) كان قد عرّف الإنسان بأنه كائن يتشبث بشبكة المعاني التي نسجها بنفسه، وليس هناك أي نشاط، حتى النشاط المادي، إلا وهو منتج في الوقت نفسه لمعاٍٍ أو رموز؛ لذلك فنحن لا نستطيع أن نفهم أي ظاهرة اجتماعية أو اقتصادية أو سياسية دون الكشف عن مُسوّغها الثقافي، فالثقافة هي نفسها التي تشكل الفائدة.

ثانياً: العلاقة بين الهوية والثقافة:

تشتبك الهوية مع الثقافة، فالثقافة التي يتم تخيلها ترسم للأفراد التابعين لها هوية مبسطة، أشبه بالأشياء والأثاث السابق التجهيز والمطلوب بتميعه بكلِّ يسر، وعندها يتم خلق جوّ من العدائية لكل ماهو أجنبي، رغم أنه لا وجود لقيمٍ تقتصر على حضارات دون غيرها، أو نطاقات ثقافية خاصة بمجتمعات معينة، فقيم "التقدم" و"الحرية" ليست حكراً على المجتمعات الغربية فحسب، وإنما هي نتاج إنساني يجب التشارك فيه؛ ذلك لأن الحضارات بأجمعها ساهمت في خلق هذه القيم وفي إنضاجها، حتى وصلت إلى الصورة التي عليها في الوقت الحالي. وإذا كان بعض الغربيين يفخر بأن الغرب يقوم على تصدير هذه القيم، فعليهم أن يعلموا أغم قاموا بتصدير قيم الدمار والنفي -أيضاً- على حد تعبير (بايار) نفسه. وإذاكانت الثقافة - كما رأيناتشكّل الحاضن للهوية، فإن الثقافة نفسها إنما هي متعلقة بالسياسة، أو بصيغة أخرى إن الثفكير الثقافي الانغلاقي يعتبر ضمنياً أن التوافق بين مجتمع سياسي ما وبين تماسكه الثقافي مسألة مفروغ منها، سواء كان ذلك التماسك الثقافي أصيلاً أو متوارثاً. فهو يعتقد أن أي ثقافة تشكل جمعاً من التمثلات الثابتة على مدى الزمن، وهو يرى أن هذا الجمع منغلق على نفسه، ويعتبر كذلك أن هذا الجمع يقرر توجهاً سياسياً محدداً؛ لذلك تنشأ نواة صلبة من التمثلات

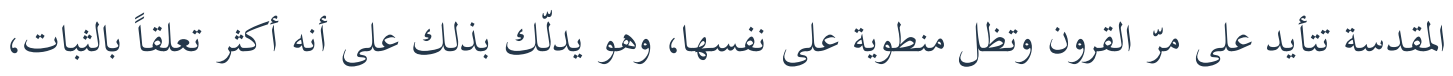
ويمتنع هذا التفكير عن استخلاص العمليات الملموسة التي تنتج عن طريقها تاريخ العناصر الاجتماعية، إنا 
تعرف نفسها في آذٍ واحد بالنسبة لتفهمها للماضي وبالنسبة لتصورها للمستقبل. ومن بين هذه العمليات الثقافية تبرز أربع منها تستحق التفصيل، وتتعلق باستراتيجيات الانفتاح على الخارج، وممارسات التحول، وعمليات التأصيل، وعمليات تكوين هويات أولية.

فالانفتاح على الثقافات الأجنبية يفترض عملية أخرى، ألا وهي نقل مغزى ممارسة ما، أو مكان، أو تمثيل، أو رمز، أو نص إلى الطرف المتلقي؛ لأن الانفتاح يكون حسب تعريفه تقريباً، إعادة تفسير واشتقاق، وإضفاء مغزى جديد على نصوص ورموز منذ آلاف السنين؛ بغية تجنيدها لخوض معارك تخدم الطرف المتلقي، إفا عملية تحويل المعاني بغية استثمارها في الواقع السياسي وبما ينصبُّ في النهاية في بجال الهوية. أما عمليات التأصيل، أو ما يسمى (بتصنيع الأصالة)، فإنها تشكل الوجه الآخر والمساعد لعمليات تحويل المعنى أو الدلالة بحيث يتم اللجوء إليها بشكل مستمر من قبل أصحاب التوجه الثقافي الانغلاقي؛ وذلك للمحافظة على النقاء الأصلي للهوية من ضروب التلوث الخارجي، ومن اعتداءات الآخرين، وحتى إن لزم الأمر يتم تحقيق ذلك من خلال مساعٍ ارتدادية. وهذا الخطاب الاستيهامي المتعلق بالماضي الذي يعاد إحياؤه بالكامل يمثل رد فعل انتقادي للحاضر الذي هو -دائماً- في موقع السلب والنفي. وانسجاماً مع ذلك تعاد عملية التعرف على الهوية، التي هي في أساسها بجرد عمليات تماهٍ مع الماضي، وعندها لا تكون الموية التي يتشدق بما أنصار الانغلاق الثقافي متواجدة إلا بشكلٍ مستقل عن المتكلمين باسمها، إنها تنعقد أو تنحل عن طريق عمليات التماهي هذه، وبذلك يعاد إنتاج هوية أي إنتاج ثقافة تعكس العلاقة مع الآخرين، كما تعكس العلاقة مع الذات، هذه العلاقة التي لا تتأثر بالزمن، والتي يلعب فيها التخيل دوراً مركزياً يتم من خلاله تكوين رؤيةٍ للعالم تحرص على إضفاء الذاتية والرجوع إلى الأصل الذي هو النقاء، والابتعاد ما أمكن عن التلوث بالآخر؛ بغية الحفاظ على طهارة المجتمع وبكارته، إنه خطاب تركب تخيلي يتحول إلى ممارسة سياسية يومية. فوظيفة التخيل المؤسسية تقوم بدور مركزي في قيام الدولة، ويف الإنتاج السياسي بوجه عام، إذا تستثمر من جانب المحرين السياسيين، فعندما يعلن رئيس الكامرون "أنا أبو الأمة، أفكر في الجميع، في كل أبنائي" فإنه يتخذ لحسابه صورة رئيس أهل البيت، مع إضفاء صبغة دينية عليها؛ لكي يجعل نفسه -ضمناً- الإله الأب، وفقاً للمفهوم الدارج في النظم التسلطية التي تسود تلك المنطقة. إنه تطويع للوعي العائلي على 
حساب السياسي، ورسم صورة تخيلية توظف من قبل السياسي الذي يصبح في النهاية رمزاً عائلياً ودينياً . وبنفس الطريقة يتم الربط بين مجالي: السياسة، والغذاء، فالأكل عملية فيزيولوجية ضرورية تفرز في الوقت نفسه لذةً شديدة، لها ارتباط صريح بالجنس، والأكل مصدر انفعالات وإحساسات بالحرمان، كما أنه يبلور -أيضاً- صفات أخلاقية يكون انتهاكها مثار فضيحة أو استهجان، وفضلاً عن ذلك فإن هذه الحاجة الضرورية الممتعة والمعنوية متبادلة؛ إذ إفا تتيح ضروباً متتازة من الأجواء الاجتماعية، وتشكل -من جهة أخرى- مرحلة رئيسة لبث الروح الاجتماعية، فالحاجة الضرورية والمتعة والمشاركة والطابع الدارج تكسب التحول في بجال الغذاء إلى التحرك السياسي نفوذاً فريداً يمكن أن يصبح مبداً يقوم على أساسه (حكم البطون)، كما في أفريقيا .

وإذا كان الغذاء يشكل علاقة ارتباطية مع المجال السياسي، فإن الملبس يشكل -بصورة مضاعفة- هذه العلاقة، إذ إن الملبس يبرز الصلات الخفية بين المادي والفكري، وبفضل هذه المرونة يشكل الملبس أداة متميزة لبناء الهويات والتعاملات بينها، إنها هويات فردية، وجماعية أيضاً، فالثوب يصنع الكاهن - كما يقول المثل الفرنسي - والمحركون السياسيون يدركون ذلك تماماً، فهو يورث القداسة والنقاء أو التلوث؛ لذلك يحرص الدكتاتوريون العصريون على الظهور في شاشة التلفزيون في بزة مكونة من ثلاث قطع، وذلك كاف لجعل نظامهم مدنياً، ولطمأنة الرأي العام. وهكذا فإن التخيل لا يمثل كلاً متماسكاً في مجتمع معين مادام يشمل سديماً من الأشكال غير متجانسة ودائمة الانفلات، فالمنتجات التخيلية ليست بالضرورة متماثلة الأشكال، فهي -بحكم تعريفها- منتجات رمزية، متعددة المعاني وملتبسة، وهي تسهم بهذه الصفة في تماسك المجتمع دون أن تتوافر أبداً إمكانية إثبات تماسك عالم معانيه، أو حتى التسليم بإمكانية ذلك الإثبات . إن التخيل الذي تمارسة السلطة السياسية على المجتمع ينبع من معرفتها أن المجتمع ليس جسماً موحداً تمارس فيه سلطة واحدة فقط، وإنما هو في الواقع بتمع وارتباط وتنسيق وتدرج -أيضاً- لسلطات مختلفة تظل مع )ذلك لكل منها خصائصها، وقد عبر عن ذلك (فوكو) بمقولته (المجتمع أرخبيل من السلطات المختلفة . 
يُعدّ (أندرسن) أبرز من درس مفهوم "الهوية المتخيلة"، إذ ينطلق من فرضيةٍ تقول: إنه لا يمكن استخلاص تعريف علمي لكلمة الهوية القومية، ومع ذلك فالظاهرة كانت وما زالت موجودة، ولكن وجودها لاحق لسياسات تخيلية خلقت فيما بعد مفهوم القومية وبلورته، إذْ هي -وفقاً لذلك- مضض اختراع، ويدلل على ذلك بالعديد من مشاهداته، لا سيما في الدول الإفريقية، إذ يُعِّرف القومية بدءاً من محدداتٍ ثلاثة: -1 الحداثة النسبية للقوميات في أعين المؤرخين، في مقابل قدمها الذاتي والخاص في أعين القوميين.

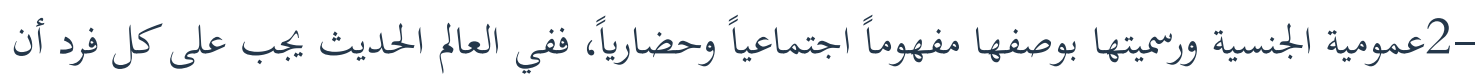
تكون له جنسية، وهذا ما ولّد مفهوم الخصوصية المتميزة. -3القوة القومية السياسية في مقابل فقرها الفلسفي وعدم اتساقها النظري، وبذلك تُعرّف القومية وفق سياقها الانتربولوجي فحسب على أهما مجتمع سياسي متخيل، وهو متخيل -أيضاً- في كونه محدد الأصول، وذا سيادة سياسية .

إن الهوية القومية إذاً مفهوٌٌ متخيل؛ لأن أبناء حتى أصغر القوميات لا يعرفون أغلب أفراد قوميتهم، ولا يقابلوغم، ولا حتى يسمعون بهم، ومع ذلك ففكرة تجمعظم وتوحدهم تعيش في ذهن كل واحد منهم، وبتعبير (آرنست غلنر) القومية ليست صحوة الأمم لإدراك الذات، إفا تخترع الأمم من حيث لا توجد . ولكن هل تخضع القومية وفقاً لذلك لنفس معيار النسق الأيديولوجي من حيث بجييشها وتوظيفها وفق الرغبات السياسية وبذلك تكون وليدة اللحظة التاريخية ولا تسبقها بأي حال من الأحوال، إن (أندرسن) ينفي هذه المقارنة بين القومية والأيديولوجيا، وإن كان لا يستبعد أن تتأسس الثانية على الأولى، ولكنه يطالب بوجوب فهم القومية بتوحيدها، ليس مع أيديولوجيات سياسية في دائرة الوعي، ولكن مع الأنظمة الحضارية السابقة عليها، والتي ظهرت من خلالها، كما ظهرت ضدها وهو لذلك يحدد دراسة القومية بفهم المجتمع الديني وحكم الأسرة. فالمجتمع الديني غالباً ما يرتبط بلغة مقدسة، ترى أها مركزية في العالم ومرتبطة بنسق قوة غير أرضي، مما يجعل اللغة أداة اندماج ديني أولاً، وتتصاعد فيما بعد لتصبح لغة قومية عن طريق الوظائف التي تؤديها اللغة، كالتواصل الاجتماعي الأفقي والعمودي، كما يؤمن تسلسل تعلم الأجيال عبر العصور، مما يجعل اللغة إرثاً تاريخياً يزيد قدسيتها وهيبتها، ولتتحول مع الزمن رمزاً قومياً تتوحد من خلاله 
الجماعات المختلفة. أما الحكم الوراثي أو حكم الأسرة فيمارس دوره في خلق القومية عن طريق توسعه أفقياً، لا سيما من خلال الزيجات الملكية التي تضم شعوباً مختلفة تحت سلطة واحدة، وبذلك تصبح هذه الشعوب خاضعة لحكم أسرة واحدة هي التي تعطي للدولة سماتما وميزاتا، ومن تئَّ قوميتها. يبدو طرح (أندرسن) هنا مختلفاً تماما عن كل التنظيرات المتقدمة المتعلقة بأصول النظرية القومية التي ترى تأسسها وصعودها مع بروز نموذج الدولة/الأمة التي شكلت قطيعة مع مفهوم الأسرة- الدولة، إلا أن (أندرسن) يرى أن تلمس مفهوم القومية لا يككن فصله أبداً عن الفترة الاستعمارية التي كانت حاضنة لنشوء القوميات والشعور بها، إذ ساعد دور النخبة كثيراً في تظهير البعد القومي لدى الدول المستعمرَة (بفتح الراء)، التي رغبت في نيل استقلالها عن طرق تنمية الحس القومي لدى أبنائها في الشعور بالخصوصية والتميز، وبذلك يكون المثقفون قد لعبوا دوراً أساسياً في نشأة القومية في الأراضي الاستعمارية، ثم لعبوا فيما بعد دور من تسلموا مقاليد الحكم، لا سيما بعد حصول هذه الدول على استقلالها؛ مما عزز لديهم الهاجس القومي، وساعد في تنميته وتصعيده. ويشير (أندرسن) إلى أن أيّ لغة استعمارية تحوي كلمات تشير إلى السكان الأصليين، كما في الإنكليزية، والبرتغالية، والفرنسية، هذه الكلمات تشير باستمرار إلى أن هناك أشخاصاً " أدنى" و"ينتمون إلى هناك" في حين تضفي اللغة الاستعمارية على نفسها صفة العلو والاستعلاء، ودور اللغة هذا ساعد على تعزيز الشعور بالاختلاف والانتماء إلى أعراق مختلفة، ومن ثَّّْ إلى قوميات متباعدة . غير أن النخبة المثقفة والمشبعة بالأفكار القومية التي لعبت دوراً حاسماً في حصول الدول على استقلالها قامت بدور الصفوة المحلية المثقفة بثقافةٍ أخرى، مما جعلها جسر الوصل بينها وبين مستعمرها السابق؛ ولذلك جرى الاعتماد عليها سياسياً، وملأت بإخلاص النسق الوظيفي المعاون في المستعمرة للشركات التجارية الكبيرة، وبقدر ما لعبت هذه النخبة دوراً سياسياً هاماً، ودوراً ثقافياً حاسماً في تعزيز القومية والانتماء إليها بقدر ما شعرت بالدونية بتحاه " اللغة الثانية" -لغة الدول المستعمرَة- مما جعلها باستمرار لا تغادر الدور الوظيفي، وتحافظ على امتيازاتا عن طريق ارتباطها بمن ناضلت ضده سابقاً وأصبحت تعمل من أجله لاحقاً. لكن إذا كانت الهوية القومية بوصفها مفهوماً قد جرى اختراعه وتكييفه، فما الذي يجعل البشر يموتون في سبيله ودفاعاً عنه؟ هنا تطرح علاقة القومية بالعنصرية، فالقوميات تفكر دائماً بأسلوب المصائر التاريخية، بينما تحلم العنصرية بتلوث أبدي ينتقل من أول الزمان، عبر توال لا غائي للزيجات الممقوتة الكريهة خارج 
التاريخ، وبفضل" فرشاة القار" سيظل الزنوج زنوجاً للأبد على حد تعبير (أندرسن) نفسه، وبفضل "بذرة

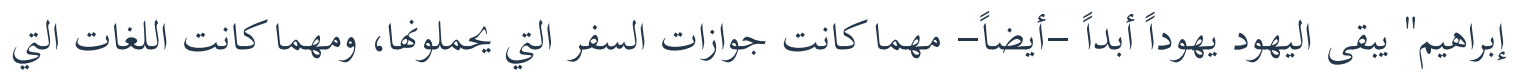
يتكلموفا ويقرؤوها، وعلى ذلك فاليهودي الألماني كان -دائماً- أفّاكاً لدى النازيّ وهذا المفهوم نفسه هو الذي ساعد على ظهور الصهيونية ونشأة إسرائيل على شكل إعادة تخيل مجتمع ديني قديم على أنه قومية من ضمن القوميات، وبذلك تمثل نشأة إسرائيل لدى الفكر اليهودي تغيراً كيميائياً من الجوّال المخلص إلى

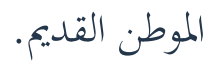

وبذلك يمكن اعتبار إسرائيل نموذجاً مثالياً على نجاح قومية شتات في اختلاق قومية خاصة بها، إذ إننا نجد اليهود أنفسهم الذي لا يشتركون لا باللغة، أو الثقافة، أو التراث، أو التاريخ، أو حتى إزاء موقف محدد من الدولة اليهودية، رغم ذلك، فإفم يسعون إلى تكوين أمة يهودية عن طريق صناعة تاريخ خاص بها، واختلاق لغة من خلال إحيائها من جديد، فأصبحت القومية الإسرائيلية التي هي عبارة عن مجمع لغات وثقافات بمثابة الهوية الاندماجية العلمانية التي يصر عليها زعماء إسرائيل المتكررون، إلا أغا -وبحكم تكوينها التاريخي - لم تلبث أن تحولت إلى ثقافة أكثر عزلة وانعزالاً. لقد ارتبطت العنصرية إذاً مع ظهور القومية وتناميها، وغالباً ما اندججت مع السيطرة الأوروبية التي أعلنت نشوء القومية الرسمية، والسيطرة الاستعمارية عن طريق تعميم السمو الموروث المولود في أفرادها، ونقل ذلك إلى الأجيال اللاحقة، فإذا كان اللوردات الإنجليز أسمى بطبيعتهم من الإنجليز العاديين الباقين، فإن ذلك لا يهم فالإنجليز العاديون لا يقلون سمواً بالنسبة للشعوب الأخرى المستعمرَة، وقد ساعد على نشأة العنصرية ضمن المنظور القومي ظهور البورجوازية ضمن الأرستقراطيين الذين حصروا ضمنهم المبادلات التجارية والرأسمالية. غير أن سياسات بناء الأمة القومية لم يقتصر على دور النخبة المثقفة ودور رجالات الأعمال فقط. وإنما ساعدت الدول الاستعمارية نفسها على بناء القومية في نفوس أبناء دولها، عن طريق تنمية الحماس القومي الشعبي الحقيقي، وعمليات زرع منظمة للأيديولوجية القومية عبر وسائل الإعلام، ونظم التعليم، والقوانين الإدارية، وغير ذلك، مما جعل القومية حقيقة أكثر من أي وقت مضى. 


\section{رابعاً: إعادة اكتشاف معنى القومية:}

وهكذا إذاً تبدو القومية كأفا طبعت القرن العشرين بختمها، فلا عجب بعد ذلك أن يُطلق عليه قرن تأجج الهويات القومية وصعودها، لقد كانت القومية بمثابة هاجس العصر الذي يجضر في كيان كل دولة، ويجثم في مخيلة أيّ جماعة لا تني تبحث عن دولتها وفقاً لتميز قوميتها؛ لذلك فالدولة القومية أشبه ما تكون بوريثة

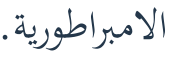

لقد حاول (آرنست غيلنر) أن ينزع عن مفهوم القومية هالته القدسية التي تكونت حوله، ونشأت من خلال أسطورة التميز والرفعة، إذ يعتبر أن القومية لا تتعدى كوها اختراعاً سياسياً، كما كان قد انتهى إلى ذلك (بندكت أندرسن) سابقاً، فالعاطفة القومية التي تثور دفاعاً عن انتهاك المبدأ القومي إنما تعبر عن سياسات التلاعب الإدراكي بعقول الجماهير ومخيلاقم؟ وهي لذلك جزءٌ من سياسات الهوية المتخيلة . ولكن هل تقتصر القومية فقط على الجماعات الممنوحة دولة؟ وبذلك لا تنشأ القومية في المجتمعات العديمة الدولة، وعندها تكون فكرة الدولة سابقة على فكرة القومية وليس العكس، فالوعي بضرورة وجود الدولة هو الذي يخلق القومية ويبرزها بعد أن كانت خفية وغير مدركة.

فالقومية كمفهوم الأمة ملتبس وعصيٌ على التحديد، ويمكن اعتباره مرتبطاً بالعصر الصناعي، إذ لم تنشأ القومية في العصر الزراعي، كما أها ترتبط بعمليات الاستعمار والامبريالية ونزعات التحرر من الاستعمار كما ذكرنا ذلك سابقاً، إذ إن ظهور المجتمع الصناعي في أوروبا لم يكن إلا نتيجة للفتح الغربي للعالم على حد تعبير (غلنر). ولكن كيف يمكن تعريف القومية وفقاً لذلك طالما أها غير ذات نسب ديني أو عرقيّ؟ هنا تعرّف القومية بأهما الثقافة المشتركة الموروثة التي تعطي الأمل في تأسيس جماعة منسجمة، وإذا كان عدد القوميات الفعالة محدوداً على مستوى العالم كله، فإن القوميات الكامنة أكثر من أن تحصى أو تُعدّ، وهكذا فالقومية تمر بمرحلة كمون قبل بروزها إلى مرحلة العلن والفعالية، وهي رغم ذلك لا يمكن اعتبارها بمثابة إيقاظ قوة قديمة كامنة سابقة، مع أها تطرح نفسها على هذه الصيغة، وإنما هي -في الواقع- ناتج أيديولوجي لاختراع مفكرين محمومين، وهي نتيجة شكل جديد من التنظيم الاجتماعي، قائم على إدماج داخلي عميق لثقافات رفيعة معتمدة على التعليم، وتحمل كل منها دولتها الخاصة بها، كما أها تستثمر بعض الثقافات الموجودة سابقاً محوّلة إياها في خدمة عملية بناء القومية. 
وعلى قدر ما توجد ثقافات متعددة ومتنوعة في العالم بحكم التمايزات الثقافية المستمرة، على قدر ما تترشح القوميات للظهور والصعود؛ لتتماهى بعد ذلك في مفهوم الأمة الذي يمثل الصياغة الثقافية لمعنى الدولة السياسي بامتياز، فما يولّد الأمم هو القومية، وليس العكس، فالقومية تستخدم تكاثر الثقافات أو الثروة الثقافية الموجودة سابقاً والموروثة تاريخياً، وتحولها -أحياناً- بصورة جذرية، كما تعمل على إحياء الثقافات الميتة، واختراع التقاليد، واستعادة النقاوات الأصلية المزينة تماماً بحيث تبدو القومية في النهاية بمثابة الخليط الخلاّق، وبعد فترة الانتقال من الكمون إلى البروز، تكف الثقافات الرفيعة التي تبقى على قيد الحياة، عن أن تكون وسيلة وعلامة مميزة لفئة مثقفة، إذ تصبح بدلاً عن ذلك وسيلة وشعاراً لأمة قومية. يضرب الأنثربولوجي الشهير (آرنست غيلنر) أمثلة على ذلك بالإسلام الذي خصص له كتباً عدة، إذ يعتبر أن الإسلام والعالم العربي نموذج واضح وجليّ على فترة بزوغ القومية؛ ذلك بأن التاريخ الثقافي للعالم الإسلامي خلال القرون الماضية يثبت حصول عمليات إصلاح إسلامي مستمرة تتقدم وتتراجع، وهي نوع من البروتستانتية الإسلامية عن طريق تأكيدها الشديد على النصوص المقدسة، والعداء الراسخ لما يسميه (غلنر): السمسرة الروحية للوسطاء المحليين بين الإنسان والله؛ لذلك فهو يعتبر أن الإسلام يستحق وصف الإصلاح الدائم بسبب تحولاته الذاتية المتتابعة من أجل الإصلاح، وأحد تحولاته تلك صادف مجيء القومية العربية الحديثة، التي لا يككن فصله عنها إلا بصعوبة عظيمة، فظهور الأمة، وانتصار حركة الإصلاح يبدوان جزأين من حركة واحدة، واستفادت الحركة القومية العربية من النزعة التوحيدية الراسخة في الإسلام، والثابتة في المجتمعات المسلمة عبر تأكيد صعودها بربط ذاها بالإسلام، فحملت بذلك تراثاً عظيماً من جهاتٍ عدة. أولها: توظيف اللغة العربية باعتبارها اللغة القومية، إضافة إلى تأجيج نزعة التحرر والجهاد من أجل التخلص من الاستعمار مما جرى توظيفه في النهاية في خانة القومية، وبذلك تمكنت القومية العربية عن طريق حملها للتراث الإسلامي من التحول إلى نزعة قوية ذات إرث عريق وثقافة رفيعة، الأمر الذي يضمن بقاءها واستمرارها.

فالقومية إذاً، كما الهوية، مضض اختراع سياسي، ولكن إذاكانت القومية تقوم على نزعة أيديولوجية سياسية، فهل من الممكن أن يجري تعديلها كما يجري للكثير من الأيديولوجيات؟ إننا نستطيع أن نتوقع أن يجري تعديل للقومية؛ ذلك بأها تنشأ وتصبح الحاجة إليها ملحة عندما تزداد الثغرة بين المجموعات السكانية 
المندجة صناعياً، والممنوحة حق الانتخاب سياسياً واقتصادياً، وكلما ضاقت هذه الثغرة فإن الشعور بها وإدراكها لا يكون على مثل هذه الحدة التي تنشأ عندما تزداد الثغرة، ولكن ذلك يطرح سؤالاً أكثر راهنية، ويتعلق بإمكانية غياب القومية وزوالها، عندما تدمج المجتمعات في دورة اقتصادية وصناعية، تغيب عندها الفوارق بينها، كما يجري الآن في ظل العولمة، وإن كانت تنتج أنماطاً أخرى من التهميش، وعندها تصبح القومية مرتبطة بالثقافة التي نشأت من ضمنها، فالثقافات الشعبية والتقاليد الصغيرة لا تبقى على قيد الحياة إلا بصورة اصطناعية، حيث تبقى في التداول بفضل جمعيات حفظ اللغة والفنون الشعبية، في حين أن الثقافات الرفيعة تتقارب مع بعضها وتتداخل بوثوق كبير، لكن دون أن تلغى فائياً، فكما أن الأمم لم تكن متشابهة في الفقر فهي لن تكون متشابهة في الرفاهية، كما يقول المثل الشهير، فالقومية سوف تكف عن أن تكون مشكلة، ولكنها ستستمر بالوجود، وتحاول التشبث بالبقاء، ولكن في شكل مخفف وأقل عنفاً على حد تعبر (غلنر).

وبنفس المعنى إذا كان مفهوم الهوية يعبر عن وهم لا وجود حقيقيّاً له، وإنما توجد استراتيجيات للهوية يتم توظيفها لخدمة هوية سياسية مصطنعة، وإذا كان عالم التخيلات والرموز موظفاً -أيضاً - لخدمة هذه الهوية المركبة، فكيف يتم إذاً الحفاظ على التمايزات؟ أو كيف يمكن جعل التنوع والاختلاف قائماً وصحياً -أي دون خوف مَرَضيّ- في نفس الوقت؟ هنا يبيء دور المجتمعات في ابتكار دورٍ مفارق للحداثة، فالحداثة تجربة اجتماعية خاصة بمرحلة معينة وخصوصاً بحربة العولمة، إها مرحلة موجهة نخو المستقبل، ومتصورة على أها ستكون على الأرجح مختلفة وأفضل إن أمكن من الحاضر والماضي. وإذا كان فوكو يعتبر أن الحداثة حالة قائمة، لا مرحلة من مراحل التاريخ، فإن السؤال الذي يجب أن تطرحه المجتمعات على نفسها هو كيف نشأت الحداثة؟ وهل في الإمكان خلق نموذج خاص بالحداثة في كل مجتمع؟ إن البديل الذي يجب أن يُطرح بدلاً من الانزواء والانغلاق، أو التماثل والتوحد، هو إعادة صياغة الفوارق، دون الحاجة إلى جعلها شرطاً مسبقاً، إذ إن هذا الإصرار يثير الريبة؛ لأنه يفتح الطريق أمام كافة أنواع القيود الذهنية والسياسية، فالخطاب الانغلاقي يجصر أكثر فأكثر المجتمعات التاريخية في تعريف جوهري لهوياتا، بإنكار حقها في الاستعارة وفي الاشتقاق الخلاّق، أي التغيير الختمل عن طريق الابتكار المفارق للحداثة. لقد بات من الملح اليوم مواجهة التيار الانغلاقي بعقلية فلسفية حديثة تفرق بين نصيبيّ كلٍ من: المحتمل والعام، 
مادام المشهد السياسي اليوم يخوض معركة الهوية. ولكن هل ارتبط تأجج الشعور بالهوية القومية مع ظهور مفهوم الأمة تاريخياً? إن علينا -بداية- أن نميز بين مفاهيم الأمة والدولة والقومية؛ ذلك بأنّ تعريف الأمة يختلط بالقومية ويلتبس بها، فالأمة ليست قديمة قدم التاريخ، حيث إن معناها الحديث لا يعود في القدم إلى أبعد من القرن الثامن عشر، ورغم ذلك فإن تطور الأمة التاريخي جعل التعاريف المقدمة، جميعها غير قادرة على الاستيفاء بالشروط التاريخية والسياسية والثقافية المنتجة لما، وإن كان تعريف (ستالين) هو الأكثر شهرة، الذي يرى فيه أن الأمة هي عبارة عن وحدة مستقرة، تطورت تاريخياً من اللغة والأرض والحياة والاقتصاد والتركيب السيكولوجي وتمظهرت فيما بعد في وحدة الثقافة. ويرى المؤرخ النمساوي الشهير (إريك هوبزباوم) أن هذا التعريف رغم شموليته التي يوحي بها يبدو نظرياً أكثر منه واقعياً، إلا إذا اعتبرنا أن جميع الكيانات التي تخرج عن إطار هذا التعريف هي مجرد استثناءات، عندها يكاد يكون الاستثناء نفسه هو القاعدة وليس كما يقولون: إنه يأتي ليؤكد القاعدة لا أن ينفيها؛ لذلك فهو يربط تعريف الأمة بالوعي الإرادي لأفرادها المنتمين إليها، فخلق الأمة يعني إرادة الأمة بأن تكون أمة، فإذا أراد عدد كاف من سكان جزيرة (وايت) أن يكونوا أمة "وايتية"، ستوجد حتماً أمة "وايتية ". وهذا ما يفرض علينا إعادة النظر في جميع التعاريف السابقة المقدمة، واتباع فج اللاأدرية موقفاً مبدئياً لدراسة حقل الأمة والقومية بشكل يمنعنا من تبني أي موقف قبلي لتشكيل الأمة، فالأمة ليست كياناً سياسياً أو اجتماعياً غير متغير، أو أها تنتمي حصراً إلى عصرٍ معين حديث تاريخياً، وإنما هي كيان اجتماعي فقط طالما أها ترتبط بنوع محدد من دولة إقليمية حديثة هي الدولة-الأمة، أما النزعة القومية التي تأخذ في بعض الأحيان شكل ثقافات موجودة سلفاً وتولها إلى أمم، فالنزعة القومية تسبق الأمم، والأمم لا تصنع الدول والقوميات، بل العكس هو الصحيح، كما أن الوعي القومي الذي يوجد لدى أفراد الأمة، ينشأ على نهو غير متكافئ بين الفئات الاجتماعية وبين الأقاليم المختلفة التابعة للدولة، وهذا ما كان يتم بحاهله تماماً في الماضي، فهناك أمثلة كثيرة تؤكد أن الدولة هي التي سبقت الأمة، كما هي حال (تركمانستان)، و(أوزباكستان)، و(كازاخستان)، ودول أخرى في آسيا الوسطى صنعت من نفسها أمحاً بإعادة كتابة تاريخها دون أن تكون نابحة عن قومية قديمة دائمة. 
لقد حاول (هوبزباوم) أن يدرس تطور النزعة القومية بين فترتين تاريخيتين محددتين، بدءاً من 1870م وحتى 1918م والفترة الأخرى التي شهدت أوج النزعة القومية بين 1918م و 1950م تم يستخلص نتائجه وانطباعاته عن النزعة القومية في أواخر القرن العشرين، فيعتبر أن الأمم والنزعة القومية لم تكن في الفترة الأولى تمثل مشكلات داخلية كبيرة، بالنسبة للكيانات السياسية التي وصلت إلى مرحلة الدول القومية مهما كانت هذه الدول متنافرة قومياً، وفق المعايير الحديثة، ذلك على الرغم من أها كانت مصدر إزعاج حادِّ للامبراطوريات اللاقومية، التي كانت غير قابلة للتصنيف تحت صفة متعددة القوميات. بل إن النزعة القومية نفسها لم تكن جاهزة في التمثيل الشعبي السياسي، كما تكشف عن ذلك عبارة (ماسيمو دازيليو) في أثناء الثورة الإيطالية ضد الهيمنة النمساوية، عندما قال: (لقد صنعنا إيطاليا، والآن علينا أن نصنع الإيطاليين)، والأمر نفسه نجده لدى المحرر النهائي لبولندا (كولونال بيلسودسكي) في عبارته: (إن الدولة هي التي تصنع الأمة وليست الأمة، هي التي تصنع الدولة).

فإذا حاولنا دراسة العوامل التي تعزز نشوء الأمة القومية وتبلورها، كاللغة، والتاريخ، والثقافة فإن (هوبزاوم) يشكك -باستمرار - في قدرقا على إنشاء القومية بشكل منفرد ومعزول عن الدور السياسي لها، كما يجري تصوير ذلك في الأدبيات الغربية في فاية القرن الثامن عشر، إذ يرى أن اللغة الأم التي يتعلمها الأطفال من الأمهات الأميّات، ويتكلموها في الاستعمال اليومي، لم تكن -بأيّ شكلٍ من الأشكال- لغة قومية، فاللغات القومية هي -بشكلٍ شبه دائم- تراكيب شبه اصطناعية، وفي بعض الأحيان تكون لغة مبتكرة كالعبرية الحميثة، فاللغة القومية هي ابتكار قياسي مُفصّح، ومكوّن من عددٍ من اللغات المحكية التي يجري تخفيضها فيما بعد إلى لهجات، وتكون المشكلة الناجمة عادةً من كوغا عبارة عن تركيبة، هي اللغة التي يتم اختيارها كأساس لهذه اللغة المفصحة والمتجانسة، فاللغة كالقومية تصطنعها أو تبتكرها الدولة، فاللغات تزداد بازدياد الدول، وليس العكس. وعندما بلغ التطور الأوروبي درجة معينة ظهرت الوحدات اللغوية والثقافية لشعوبٍ نَضَجَتْ بصمت على مَرِّ القرون، أصبحت واعية لنفسها بوصفها قوة ذات مصير تاريخي، فصارت تطالب بالسيطرة على الدولة بوصفها أعلى أداة متاحة للسلطة، وتناضل لتقرير مصيرها الذاتي السياسي، فتأريخ ميلاد المشروع السياسي للأمة كما يرى (هوبزباوم) هو عام 1789م أي: عام الثورة الفرنسية ورفع شعار دولة واحدة فقط للأمة 
بكاملها، الذي يختصر المشروع القومي ومطالبه في نشأة الأمة وتشكيلها، إلا أن اللحظة التاريخية التي تحول فيها مفهوم الأمة إلى مبدأ القومية كانت في فاية الحرب العالمية الأولى، الذي عنى بشكلٍ غير مقصود اهيار الامبراطوريات الكبرى المتعددة القوميات في وسط أوروبا، وحدوث الثورة الروسية التي ظهرت لوصفها ثورة اجتماعية أكثر مما هي تقرير لمصير قومي، نشأ عندها ما يسمى بالمسألة القومية، لاسيما مع معاهدة (فرساي)، التي أعادت رسم الخارطة السياسية لأوروبا على أسس قومية، وأصبحت الدول الآن فوق الحدد المتوسط بدلاً من كوغا دولاً صغرى، وصارت "الشعوب المضطهدة في داخلها تدعى أقلياتٍ مضطهدة، " وكانت النتيجة المنطقية لمحاولة خلق قارة مُقسمة بدقة إلى دول إقليمية متماسكة، كلُّ واحدة كان يقطنها سكان منفصلون متجانسون لغوياً هي الترحيل الجماعي، أو الإبادة للأقليات، وهنا ظهر المنزع الإجرامي والعنصري للنزعة القومية، وازداد حضوره مع الحركات الفاشية واليمينية الأخرى التي عملت على استغلال النزعة القومية على أشدها، فخلفت وراءها كوارث بشرية تحتاج البشرية معها إلى قرون حتى تتعافن منها. لكن السؤال كيف تأثرت مصائر النزعة القومية خارج المناطق الجغرافية التي ظهرت فيها في آسيا والبلدان الإسلامية؟ هنا يجيب (هوبزباوم) مرةً أخرى بأن إعادة الاكتشاف الثقافي للقوميات والحضارات من قبل هذه الشعوب هو الذي عزّز المنزع القومي لديها، وحرّضها للحصول على دول متحررة ذات بحانس قومي بكيث أصبحت الدولة - الأمة الهدف الذي تسعى جميع القوميات إلى تحقيقه واقعاً بعد أن داعب خيالها السياسي طويلاً، لاسيما أثناء فترة الاستعمار . أما الآن، وبعد أن هدأت الفورة القومية إلى حد كبير، وانحسر دور الدولة القومية وتقهقر فإن ذلك يرجح الاحتمال القائم بأن التاريخ والثقافة القومية لن يكونا بارزين، بمعنى أغما سيمارسان أدوراً ثانوية، لكنهما لن يلعبا دوراً تحويلياً في حياة الشعوب والبلدان وسينحسر دور القومية لتكون مجرد طريقة لوصف هويات أناس يوصفون بأهم إنكليز أو (فرنسيين)، ولكن من بين هويات أخرى

يستعملوها لنفس الغرض، وهكذا ينتهي (هوبزباوم) إلى نتيجة معاكسة تماماً لما يروج له (هنتنغنتون) في مقولته عن صدام الحضارات القائمة على أساس أن الصراع القائم هو بين الحضارات، على اعتبار أن هذه الحضارات هي هويات أو كيانات ثقافية. خامساً: نهاية الهوية القومية أم غهاية "الدولة-الأمة": يستأثر الجدل النظري والفكري حول مصائر الهوية القومية أو الدولة - الأمة وتحولاثما، بمعظم النقاش داخل 
دوائر علم السياسة والباحثين في العلاقات الدولية المعاصرة، فمفهوم الدولة الذي طبع التطور السياسي لمعظم المجتمعات الغربية والعالمثالثية، ومنه استمدت العلاقات الدولية مسماها يبدو اليوم موضع امتحان عسير واختلافٍ أيدولوجي وسياسي وفكريّ عميق، فمنذ معاهدة وستفاليا عام 1648 التي وضعت فايةً لحرب الثلاثين عاماً بين فرنسا والنمسا، وجرى عندها تحديد غائيّ وترسيم كليّ للحدود بين الدول، عندها أخذ يتبلور المفهوم الحديث للدولة من حيث هي تنظيم سياسي اجتماعي للأفراد القاطنين في بقعة جغرافية محددة، ومع بزوغ النزعات القومية في منتصف القرن التاسع عشر وتحولها في القرن العشرين إلى نزعاتٍ مهيمنة، انتهت إلى نشوء دولٍ قومية في كلٍ من ألمانيا وفرنسا كما ذكرنا، فإن المأزق الأيديولوجي والسياسي والاقتصادي الذي آلت إليه هذه الدول بعد الحرب العالمية الثانية، ومن ثمّ التحولات الديمغرافية من خلال الهجرات الشرعية وغير الشرعية، دفع نخبها إلى التفكير من جديد في طبيعة تكوين الدولة القومية وآلية عملها، وهو ما يُصار إلى التعبير عنه حالياً بنهاية الدولة القومية وبداية التفكير بالدولة الديمقراطية، أو دولة

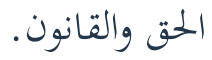

إن (جاك باغنار) -على سبيل المثال- يؤكد على تاريخية مفهوم الدولة، فما دام هذا المفهوم نتاجاً تاريخياً فإن زواله -أيضاً- مربوط بالتحولات التاريخية والاجتماعية التي تعيشها المجتمعات، فالدولة بالنسبة إليه كانت نتاجاً تطويرياً للسلطة المتصلة باجتماع ثلاثة ثوابت، هي: المأسسة الفعّالة للسلطة، والمركزة الحقيقية الصحيحة للإكراه، وتخصيص العوامل المضطلعة بمصير جماعي، وقد ارتبط تطور مفهوم الدولة، أو بالأصح ولد من رحم النموذج الأوروبي، ثم عرف تطعيمات مختلفة من هنا وهناك، بحسب الخصوصيات الأصلية لكل مجتمع من المجتمعات؛ وهو لذلك يؤكد -بعكس الكثيرين من الباحثين الغربين- أن كل دولة تمتلك هوية تكوينية خاصة ونظاماً (D.N.A) نوعياً، حتى لو بقي الحد النوعي الجنسي للدولة مُعتمداً عموماً . بعد ذلك ظهرت الصيغة الحديثة للدولة التي تعممت ودّياً عن طريق القوة في أوروبا خلال القرون الوسطى، وهناك تمَّ ابتكار النموذج المعمّم للدولة -الأمة، ويعود الفضل في إنجاز هذه الدولة إلى ارتقاء السيادة الداخلية للحاكم الذي فرض السلطة على رعاياه؛ لأنه قبل تنظيم الشؤون الخارجية والحرب والدفاع، ثم تنظيم

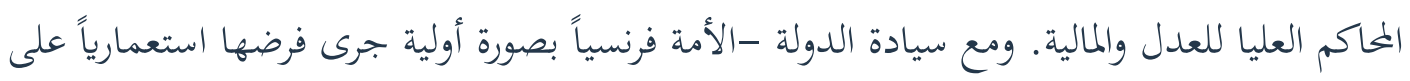
مجتمعات لم تكن مهيأة للتكيف، حيث كان استقلالما طعماً غريباً عن تقاليدها وطرائق حياتا، وهنا نشأت 
مشاكلها الداخلية و الخارجية، فحدود هذه الدول رسمت اعتباطياً من قبل الدول الاستعمارية التي كانت مهمومة أكثر باسترجاع بتحاراها، وبالإمساك بالنظام أكثر من احترام الهويات العرقية المتفسخة والمنقسمة بين سيادات دولية مختلفة، أما مشاكلها الداخلية فقد ابتدأت من تكويناتا الاثنية المتباينة والمثنافرة، ونخبها ذات المطامح المتفاوتة، بحيث بقيت الدولة- الأمة في العالم الثالث دولة رخوة غير مطبوعة بطابعٍٍ قانوني أو مؤسَّيّ، وانطبق عليها توصيف (ماكس فيبر) الدقيق بأن الدولة لم تعنِ سوى الاحتكار الشرعي لممارسة

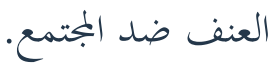
إذا كان هذا الشق التاريخي هو الذي يسيطر على الكثير من الباحثين لدى النظر في تاريخ الدولة -الأمة فإن معرفة طبيعة ممارسة الدولة- الأمة لسلطتها ولسيادتا يكشف -أيضاً- عن خلل بنيويّ عميق في طبيعة تركيبها ويظهر ذلك جلياً عند ادعاء تحقيق المصلحة العامة في قرارتا السيادية، إذ تظهر المصلحة العامة عندها كأهما أشبه بالمعطف الأيديولوجي، الذي ترتديه الدولة مؤقتاً لإثبات سيادتا، وذلك كي تحتج عند اللزوم بداعي المصلحة العليا المتأصلة بدون جدوى لتسويغ أفعالها، ويتبقى دين الدولة بعد زوال الأزمة بوصفه ديناً ممارسة السلطة المحصنة.

وإذا استعرضنا الآن العوامل التي أسهمت في إضعاف الدولة - الأمة حديثاً، فإن أهم هذه العوامل يكمن في العولمة التي اختصرت دور الدولة وأفرغت نموذج المركزة السياسية والاقتصادية الذي كانت تفرضه الدولة، كما أن انخسار دور الدولة بوصفها، شخصية قانونية فاعلة ارتبط مع بروز القوى تحت القومية، والقوى العابرة للقومية كفاعلين جدد ومؤثرين باتحاه عملية الحسم في العلاقات الدولية، وبروز معايير أو قيم جديدة مستحدثة في عملية التوازن أو الشراكة الدولية، ويضاف إلى ذلك دخول عوامل أخرى، مثل: امبراطوريات الشركات المتعددة الجنسيات، والمنظمات غير الحكومية، وظاهرة انتقال الأفراد بشكل هجرات شرعية وغير شرعية، كل ذلك وضع مفهوم الدولة ودورها في مهب الريح؛ مما دفع بعض الدول للرد على هذه التحديات بالاتحاه نحو التجمع بوصفه أفضل رد ملائم على اتساع الرهان والمجازفة؛ لذلك أصبحت روابط الدول متعددة ومتنوعة في جميع القارات، فتشكلت (ALENA) التي ركزت على مصالح كندا، والولايات المتحدة، والمكسيك، وأيضاً الآسيان (ASEAN) التي ضمت بروناي، وأندونيسيا، وماليزيا، وسنغافورة، وتايلاند، وغيرها من التجمعات الإقليمية ذات المصالح المتعددة كالاتحاد الأوروبي. 
لكن ظهور مثل هذه التجمعات الإقليمية لا يخفي حقيقة التحديات المصيرية التي باتت تقف أمام الدولة ودورها السياسي؛ ذلك بأن تحولات سياسية وجغرافية وأيديولوجية جديدة بدءاً من سقوط الاتحاد السوفيتي

وما تلاه من تفككٍٍ لدول المنظومة الاشتراكية، وما تبع ذلك من انبعاث الهويات القومية، الذي أدى إلى ظهور دول جديدة على الساحة الدولية، التي حملت معها صراعاتها الكائنة في جذور تأسيسها، بجكم كون حدودها كانت -وما تزال- تمثل بؤراً مثيرة للتوتر باستمرار، يضاف إلى ذلك بروز دور خجول لمنظمة الأمم المتحدة بعد غياهما المستمر على مدى عقود، والذي فرضته ظروف الحرب الباردة، وقد ظهر هذا الدور بداية في حرب الخليج الثانية حينما حشدت الولايات المتحدة قوات حلفائها تحت اسم الأمم المتحدة، وكانت مهتمة في إصدار قانون عن بجلس الأمن يسمح بإخراج القوات العراقية من الكويت بداية، ويفرض العقوبات على العراق فيما بعد. لقد مكن هذا الغطاء الدولي الولايات المتحدة من ممارسة أهدافها بتحاه النظام العراقي، وبدأ عندها يتصاعد ويبرز دور جديد للأمم المتحدة لطالما جرى تغييبه، بل واللعب فيه، حتى بدت الأمم المتحدة كأفا تلعب دور شاهد الزور على الشرعية الدولية، وتحلى ذلك عندما قام حلف (الناتو) بالضربات الجوية على (صربيا)؛ مما أثار أسئلة كثيرة عن دور الأمم المتحدة، وقامت موجة حادة من النقد للولايات المتحدة لعدم قيامها بالتنسيق مع دور الأمم المتحدة، وقد عدّ البعض حينها أن ظهور مثل هذه

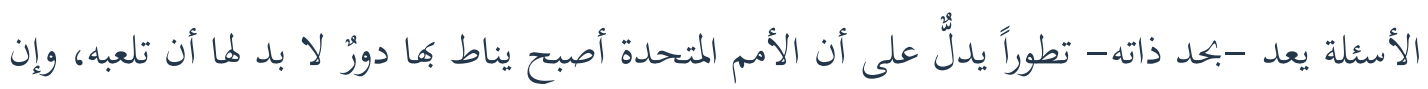
تقاعست عنه، أو لم تتمكن من أدائه. وهذا ما ساعد -أيضاً- على بروز مفهوم جديد للعلاقات الدولية يدور حول المشكلات المثارة في إطار البيئة، ومحاولة حصر الخراب الناجم عن التقدم التقني، والخوف من تبديد الثروات الزراعية والحيوانية الطبيعية،

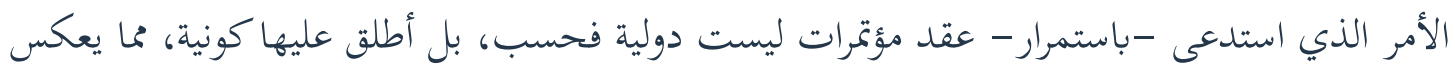
التزاماً معنوياً وأدبياً بتحاه الدول جميعها ومسؤولياها في تفاقم هذه الأزمة البيئية. يضاف إلى ذلك سيادة مبدأ قانون السوق الذي هو قناع (الليبرالية)، حيث أصبح بمثابة الطريق الوحيد، وغابت كل البدائل الاقتصادية التي تمتلك القدرة على المنافسة، وهذا فرض طرح تأثيرات العولمة اقتصادياً، واجتماعياً، وحتى سياسياً على سئل دول الأطراف أو الجنوب التي غالباً ما تكون ضمن خانة الإقصاء والتهميش، وتتلقى التأثيرات السلبية لكل التحولات والتغيرات العالمية والدولية. 
إن كل ذلك فرض حقيقة جديدة تتعلق بالطريقة التي تدار بها الأزمات الدولية، وهذا ما فسح البمال للأمم المتحدة لكي تمارس أدواراً غالباً ما تم تغيببها أو تمميشها، وأصبحت تلعب الآن دوراً رئيساً على الساحة، بل ويطلب منها الحضور على مسرح العمليات في كل مكان تقريباً (يوغوسلافيا، والصومال، والعراق) ولكن حظها من النجاح جاء متبايناً، سواء في أزمة (كوسوفو) أو في (تيمور الشرقية)، أو الحرب الدولية على الإرهاب، ثم الأزمة العراقية، وغير ذلك، ومهما يكن مقياس النجاح والإخفاق فإن كل الأحداث أو الأزمات التي حدثت في العقد السابق تكشف قراءقا عن حدوث منعطف جديد في أسلوب إدارة الأمن الدولي، فبدلاً من النظام القائم على مصفوفة من الدول المتساوية في السيادة، يُطرح الآن نظام بديل يقوم على حق المجتمع الدولي -وربما واجبه- في التدخل حيثما يوجد انتهاك. متعمد للحقوق الأساسية للجماعات البشرية، حتى ولو كانت السلطة القائمة هي المسؤولة عن هذا الانتهاك، من ناحية أخرى يبدو العالم مهتماً أيضاً بمطاردة المسؤولين عن الإبادة، وعن الانتهاكات الأخرى للحقوق الإنسانية وتقديمهم إلى المحاكمة (إنشاء المحكمة الجزائية الدولية بموجب اتفاقية روما لعام 1998). وبهذه الطريقة يتجدد الأمل في وضع الأساس لنظام عالمي موره القانون .

ولكن السؤال الذي يطرح نفسه، وهو : هل معنى ذلك أننا بصدد مولد نظام دولي جديد وحقيقي هذه المرة؟ قد تكون النوايا حسنة في ذلك، إلا أن العقبات التي تواجه تحقق ذلك تكاد تتغلب على القراءة المتفائلة للأحداث الدولية، وأولى هذه العقبات هي النظر إلى التجاوز أو الخرق الذي مارسته الولايات المتحدة بحربها على العراق دون الحصول على قرار دولي من الأمم المتحدة. مهما يكن فالدول القومية أصبحت إذاً تتخبط تحت وطأة تناقضاتما الداخلية، لا سيما قوى الظل المتمثلة في التنظيمات الخفية أو السرية التي تخترق المجتمعات من داخلها، وتحاول وضع يدها على مراكز العصب الحساسة في أجهزة صنع القرار، مثل: عصابات المافيا، أو القوى الأجنبية المتدخلة في الشؤون الداخلية للدول، يضاف إلى ذلك دور الجماعات الخفية من المضاربين التي أصبحت تتلاعب بالاقتصاديات الوطنية، وأصبح بمقدورهم الخاذ قرارات تؤدي إلى بناء ثروات الأمم أو هدمها؛ ولذلك أطلقت الصحافة الإنكليزية عليها لقب: (الأولاد الذهبيون .Golden boys) (يضاف إلى ذلك -أيضاً- أن الدول قد أصبحت معرضة بشكل متزايد لضغوط خارجية أكثر تنوعاً وأشد وطأة، لكن ذلك لا يعني أن الدولة قد تحولت إلى 
أداة بالية، أو إلى فاعلٍ مصيره الفناء، على حد تعبير (ميرل)؛ ذلك بأن قدرة الدول القومية على البقاء، لا تفسرها عوامل تتعلق بالعقلانية النظرية، بقدر ما تفسرها عوامل تاريخية وثقافية، فتصاعد دور الهجرة التي راكمت دور التحولات الديمغرافية، وفرض تغيرات سكانية أصبحت بمثابة فاعل رئيسي في العلاقات بين الدول التي تعززت مع تطور التقنية وبروز الثورة الاتصالية؛ ولذلك تبقى القيم النظرية عاملاً يمارس ضغطاً معنوياً -باستمرار - على الدول، لا سيما التزامها بقيم الديمقراطية وحقوق الإنسان، ومساعداً لبروز قيم عالمية جديدة، إلا أن ما يعيق ذلك ويمنعه هو التلاعب الدولي المستمر بهذه المفاهيم على طريقة الكيل بمكيالين في تطبيق هذه القيم؛ مما يجعل الحلم الكامن في تحقيق ديمقراطية العلاقات الدولية نوعاً من السراب لا سيما أن المفاوضين الاجتماعيين الذين لا يمثلون الحكومة ويمثلون ما يسمى المجتمع المدني، لم يمثلكوا بعد الشرعية القانونية في ممارسة قدرٍ من النفوذ الفعلي، ويبقى دورهم نظرياً أكثر مما هو قابل للتحقق عملياً، وبذلك تبقى الدولة هي الحجر الرئيسي الذي يعوّل عليه في الانتقال منه إلى تحقيق ديمقراطية العلاقات الدولية، فهيئات المجتمع المدني ومثثلوه عليهم أن لا ينسوا -باستمرار - إفم رعايا دول قبل أن يكونوا مواطنين عالميين، وأن الدول ما تزال ركناً أساسياً لا يمكن الاستغناء عنه لانتظام العلاقات الدولية. يمكن القول إذاً إن سؤال الهوية يبدو اليوم أكثر راهنية وحضوراً مع تزايد وتيرة العولمة بتجلياتا الاقتصادية، والإعلامية، والمالية، وحتى الأمنية، وهو ما يطرح تحدياً حقيقاً على المجتمعات العالمية عموماً، والعربية والإسلامية خصوصاً؛ لأن نسبة إسهامها في حركية العولمة وتدفقاهما هي الأقل، من ثَّهّ هي الأكثر عرضة لانعكاسات العولمة وآثارها السلبية؛ ولذلك يبدو من الضروري اليوم دراسة مفهوم الهوية وفق نظرة ورؤية جديدة، تنفتح بابتحاه المستقبل أكثر من انغلاقها على الماضي، وهو ما يوجهنا قدماً باتحاه بلورة رؤية جديدة لمفهوم الهوية في إطار المجتمعات الإسلامية. 